https://www.sciencedirect.com/science/article/pii/S0959438817300946?via\%3Dihub

\title{
Reprogramming Neurodegeneration in the Big Data Era
}

\author{
Lujia Zhou ${ }^{1,2}$, Patrik Verstreken ${ }^{1,2}$
}

${ }^{1}$ VIB Center for Brain \& Disease Research, Leuven 3000, Belgium.

${ }^{2}$ Department of Neurosciences, KU Leuven, 3000 Leuven, Belgium.

Corresponding authors: Lujia Zhou (Lujia.zhou@kuleuven.vib.be) and Patrik Verstreken

(Patrik.Verstreken@kuleuven.vib.be)

\begin{abstract}
Recent genome-wide association studies (GWAS) have identified numerous genetic risk variants for lateonset Alzheimer's disease (AD) and Parkinson's disease (PD). However, deciphering the functional consequences of GWAS data is challenging due to a lack of reliable model systems to study the genetic variants that are often of low penetrance and non-coding identities. Pluripotent stem cell (PSC) technologies offer unprecedented opportunities for molecular phenotyping of GWAS variants in human neurons and microglia. Moreover, rapid technological advances in whole-genome RNA-sequencing and epigenome mapping fuel comprehensive and unbiased investigations of molecular alterations in PSCderived disease models. Here, we review and discuss how integrated studies that utilize PSC technologies and genome-wide approaches may bring new mechanistic insight into the pathogenesis of AD and PD.
\end{abstract}

\section{Introduction}

Alzheimer's disease (AD) and Parkinson's disease (PD) are the most common forms of neurodegenerative disease, currently without effective treatments. $A D$ is pathologically defined by accumulation of $A \beta$ plaques, neurofibrillary tangles, and severe neuronal loss [1]. Whereas PD is often characterized by deposition of $\alpha$-synuclein and loss of dopaminergic (DA) neurons in the midbrain substantia nigra as well as other neurons in the brain [2]. A subgroup of AD and PD cases are early-onset familial forms caused by rare gene mutations, but the vast majority (>90-95\%) of clinical cases are late-onset sporadic forms. These may originate from genetic susceptibility in combination with enigmatic environmental factors. Recent large scale GWAS and meta-analyses have identified a large number of single nucleotide polymorphisms (SNPs) located in 22 loci associated with sporadic AD (SAD), and 26 loci associated with sporadic PD (sPD) $[3,4]$. The current challenge confronting biomedical researchers is to decipher the mounting genetic information as well as to pinpoint the molecular mechanisms by which the genetic variants contribute to disease pathogenesis. Reprogramming of patient-derived somatic cells into induced pluripotent stem cells (iPSCs), as well as gene-editing of human embryonic stem cells (hESCs) provides unique platforms for interrogating GWAS risk variants in human neurons and other cell types that reside in the brain. Furthermore, PSC-derived disease models facilitate genome-wide transcriptome and epigenome analyses 
to identify common molecular alterations and causal regulators in the development of complex neurodegenerative diseases including AD and PD (Figure 1). 


\section{Interrogating Risk Variants from GWAS \\ Non-coding variants in human neurons}

The vast majority (98.5\%) of the human genome are non-coding regions [5], which contain functional sequences including cis-elements that regulate gene expression. The genetic variants identified in GWAS are enriched in cis-regulatory elements [6], implicating cis-acting effects as a common factor in human diseases. In the case of SAD and SPD, the most strongly associated SNPs identified from GWAS are all located in non-coding regions. These SNPs are also often present in both patients and unaffected individuals, albeit with different frequencies. The low penetrance of GWAS risk variants also predicts their weak phenotypes. Approaches of generating transgenic animals that model coding mutations known to deterministically cause familial forms of disease, are difficult to deploy when studying the effect of GWASidentified SNPs associated with sporadic cases. Recently, human PSC-derived neuronal models have emerged as an attractive strategy for deciphering functional effects of GWAS-identified genetic variants.

Excellent examples of such strategies are seeing the light: Young et al. applied patient iPSC to dissect the molecular phenotypes of common non-coding risk variants in the SORL1 locus associated with SAD [7]. SORL1 is an endocytic receptor involved in APP trafficking and subsequently affecting A $\beta$ generation $[8,9]$. Reduced SORL1 expression was observed in the brains of SAD patients [10], but whether and how common risk variants affect SORL1 expression is unknown. To address these questions, Young et al. generated a cohort of 13 iPSC lines from 6 control individuals and 7 SAD patients harboring common variants in the $5^{\prime}$ introns of SORL1. In iPSC-derived neurons, all protective alleles (from 4 controls and 5 SADs) significantly increased BDNF-induced SORL1 gene expression, whereas all homozygous risk variants (from 2 controls and 2 SADs) had no effect on BDNF-induced SORL1 gene expression. Therefore, the detected gene-specific effects were consistent with the protective or risk haplotype of the SNPs, but were largely dissociated from the actual disease status, which are often an outcome of complex genetic and environmental factors. The study could be strengthened by applying statistical criteria to determine sample sizes taking into account the number of protective and risk alleles included in both $A D$ and control cases. As increased SORL1 expression correlates to lower $A \beta$ levels [7-9], the current study provides a possible functional link between SORL1 risk haplotypes to AD pathogenesis.

Another study by Soldner et al. used efficient gene-editing based on CRISPR/Cas9 in hESCs to assess the effects of PD-associated non-coding risk variants in the $\alpha$-synuclein (SNCA) locus [11]. They first prioritized risk variants that are located in distal enhancers based on epigenetic annotation. They subsequently introduced selected risk alleles into the genome of hESCs. In ESCs-derived human neurons, one high-risk allele increases SNCA expression by $10 \%$ [11]. Consistently, this risk allele impairs binding of sequencespecific transcription factors to its nearby DNA [11]. Given that $50 \%$ increase of SNCA causes a familial form of PD, a modest upregulation of SNCA over lifetime may represent a possible molecular basis of increased risk of PD.

There are two major challenges for future attempts to use non-coding GWAS variants for investigating disease mechanisms. The first challenge is prioritizing SNPs for functional studies, since typically a linkage disequilibrium (LD) block identified by GWAS contains multiple SNPs, with some or many among them being neutral mutations [12]. Therefore, prioritizing non-coding variants based on existing biological databases is probably crucial for successful identification of true disease-associated variants in the following functional studies. As shown by the work of Soldner et al. [11], current available epigenetic data (http://www.roadmapepigenomics.org) [13] was successfully used to filter PD associated SNPs in the 
SNCA locus based on cumulative overlap with enhancer-associated markers. Overall, advances in systematic annotations of non-coding genomes are expected to help predicting hotspots where functional SNPs are more likely located[12].

The second challenge is the small effect sizes for most GWAS alleles, which implicate relatively low penetrance and weak disease phenotypes. Instead of directly probing disease-phenotypes in patient iPSCderived cultures, one could consider that specific assays based on rational designs targeting at candidate genes are more likely to yield reliable information on the functional effects of the GWAS alleles. Take the work of Young et al, [10] for example, the same haplotypes of SNPs in the SORL1 locus are highly consistent in their effects on induced SORL1 gene expression, despite the fact that these SNPs have very low penetrance in association with disease phenotypes. Therefore, human iPSC-derived cultures allow the determination of gene-specific effects of non-coding variants as the first step, which should be followed up by studies exploring the involvement of candidate genes (which the SNPs are located within or flanking) in disease pathogenesis. Although finding the functional consequences of SNPs in cell-based assays does not prove causality of the SNPs to disease, the more relevant approach is to apply information generated from GWAS variants to guide the identification of specific genes and pathways, which are likely involved in disease pathology and therefore could potentially be targeted for therapeutic discovery.

\section{Risk genes and human microglia}

Microglia are the resident immune cells in the brain, which are activated in response to neuropathological factors and regulate neuroinflammation in neurodegenerative diseases [14]. Although microgliamediated neuroinflammation has long been recognized as a hallmark feature of both AD and PD, evidence suggesting a crucial role of microglia in disease pathogenesis has only emerged recently. Network-based analysis of whole-genome transcriptome profiling data from thousands of autopsied brain tissues from SAD patients has identified microglial pathways as the most prominent causal regulator in SAD [15]. In concordance with this finding, GWAS has uncovered mutations of several immune-related genes, including TREM2, CD33, CR1, EPHA1, ABCA7, SHIP1, APOE, HLA-DRB5-HLA-DRB1, MS4A6A, increasing the risk to develop SAD $[3,16]$. Further dissecting these GWAS variants will bring essential mechanistic insights to the role of microglia in AD pathogenesis. However, studies of microglia in human disease has been hampered by lack of relevant model systems. Primary human microglia differ substantially from rodent microglia, and protocols for reliably generating such cells in vitro are not mainstream.

Recent studies from three independent groups are starting to open this door and show that is it possible to differentiate human iPSCs and ESCs into microglia [17-19]. The reported protocols are largely similar in that they all differentiate PSCs through a myeloid intermediate, and use colony-stimulating factor (CSF1) and interleukins (IL-34) to induce microglial cell fates. These protocols produce similarly high yields of microglia, which faithfully resemble their in vivo counterparts isolated from human brain in terms of phenotypic and gene expression profiles [17-19]. Notably, these work also show that it is possible to generate $3 \mathrm{D}$ brain organoids by integrating human microglia into a cortical-like matrix of PSC-derived neurons, astrocytes and oligodendrocytes. In the context of 3D organoids, microglia show rapid responses to neuronal injury similar to brain microglia $[17,19]$. The current availability of systems to generate human microglia from PSCs, along with rapid technical maturation of 3D organotypic cultures [20] holds great promise and will likely revolutionize studies that investigate microglia and their interactions with neurons and astrocytes in human disease pathogenesis. 


\section{Unveiling common pathways via transcriptome profiling}

Genetic studies have identified a wide range of mutations that can either cause or increase risk for AD and PD. Despite their divergent genetic origins, AD or PD patients share a similar spectrum of clinical features and pathological manifestations, implicating that some common downstream pathways may contribute to the characteristic phenotypes of these diseases. Several studies of familial and sporadic patient iPSCderived neurons have captured common disease-related phenotypes in genetically diverse AD or PD cases [21-25], providing a basis for using iPSC-derived neuronal models to dissect molecular pathways involved in disease pathogenesis. Recent work has resorted to whole-genome RNA-sequencing (RNA-seq) to uncover gene networks that are disturbed in patient iPSC-derived neurons.

In an effort to explore common molecular pathologies of different genetic backgrounds in PD, Lin et al. used a collection of iPSC lines from monogenic PD patients carrying distinct mutations, including LRRK2G2019S, SNCA triplication or PARKIN-255delA, together with a sporadic PD case and healthy control individuals [21]. A comprehensive transcriptome analysis of iPSC-derived DA neurons identified hundreds of genes that are consistently deregulated in iPSC-derived DA neurons across these different PD mutations. These deregulated genes fall into major pathways including oxidative stress and synaptic transmission, which may explain related phenotypes, including increased oxidative stress susceptibility and impaired neuronal activity observed in models of PD and iPSC-derived neurons from PD patients [21]. Among the most consistently upregulated genes in iPSC-derived DA neurons from PD patients is RBFOX1, a splicing factor which regulates neuronal splicing networks and has been linked to various neurological diseases [21]. However, this work did not assess the functional impact of RBFOX1 knockdown in PD neurons and the relevance of RBFOX1 to PD-related phenotypes remains unclear. Future work should also analyze perturbed pathways and try to identify upstream genes that occupy higher hierarchical positions in the gene networks and regulate expression of downstream genes $[26,27]$. Subsequent work can then assess their contribution to disease-related phenotypes.

Other studies have also assessed the expression profile of iPSC-derived DA neurons from PD patients carrying LRRK2-G2019S mutation by RNA-seq $[28,29]$. The earlier study by Reinhardt et al. employed isogenic controls where the pathogenic mutation was corrected and identified a group of genes that are deregulated in LRRK2-G2019S neurons. Knockdown of some of the upregulated genes, including CPNE8, CADPS2, and MAP7 ameliorated neurodegeneration of LRRK2-G2019S DA neurons in vitro [28]. Another study by Sandor et al. has resorted to the purification of iPSC-derived DA neurons, as to enrich for this one cell type for RNA-seq analysis [29]. Comparing gene expression profiles of LRRK2-G2019S mutant DA neurons with those of DA neurons from healthy control individuals uncovered 168 differentially expressed genes, which functionally converge onto four major pathways including oxidative stress, glycosaminoglycans, immunity NF-kB signaling and lipopolysaccharides. Notably, the gene expression profiles of purified iPSC-derived DA neurons highly resembles that those of their counterparts from postmortem brains, validating the use of the in vitro differentiated and purified DA neurons for studying transcriptomic changes in PD. Curiously, the transcriptomic effects of LRRK2-G2019S backgrounds are similar to those of rotenone, a neurotoxin that blocks mitochondrial function [30,31], suggesting LRRK2 and rotenone may share pathogenic mechanisms. Moreover, the transcriptomic effects detected in LRRK2-G2019S DA neurons anti-correlate with transcriptomic responses induced by clioquinol, a compound shown to prevent DA neuronal loss as well as revert PD symptoms in certain rodent models $[32,33]$. These data suggest that there may be some common molecular alterations in various genetic PD 
backgrounds, and they highlight the potential of using iPSC-derived neurons for identifying and characterizing therapeutics for PD.

\section{Mapping epigenetic alterations}

Epigenetics refers to changes in gene expression caused by modifications of DNA molecules or their packaging proteins without changing the DNA sequence [34]. For complex diseases, including AD and PD, which often lack causative genetic determinants, exploration of epigenetic alterations is thought to be particularly important and may explain at least in part the environmental impact on neurodegenerative disease [35]. Recent genome-wide DNA methylation analyses using postmortem tissues have revealed epigenetic alterations in $A D$ and PD patient brains, suggesting that epigenetic changes occur in the context of these neurodegenerative disorders [36-38]. However, postmortem epigenetic analysis using brain tissues composed of mixed brain cells may underestimate or confound disease-associated epigenetic changes, as different cell populations are known to have distinct methylation landscapes [39]. In particular, the loss of neurons in AD and PD may confound postmortem epigenetic analysis by changes in the proportion of cell types. Alternatively, patient iPSC-derived neuronal cultures, along with techniques developed for purification of disease-specific subtype of neurons $[29,40]$, could represent a new avenue for interrogating epigenetic changes associated with early disease status.

Recent work shows a genome-wide DNA methylation profiling of iPSC-derived DA neurons from monogenic PD patients carrying LRRK2-G2019S mutation, SPD patients and healthy control individuals [41]. Interestingly, LRRK2-G2019S and SPD DA neurons show similar changes in DNA methylation patterns, with abnormal methylation prominently hitting enhancer elements and transcription factor (TF) binding sites, consequently up- or down- regulating gene expression. A significant correlation between changes in DNA methylation and gene expression was confirmed by microarray-based transcriptome analysis. In total, 23 TF-binding sites were disrupted by differential DNA methylation in PD neurons. Among them, several TFs including FOXA1, NR3C1, HNF4A and FOSL2 have been previously associated with the maintenance of midbrain DA neurons and with PD symptoms [42-46]. Notably, DNA methylation changes occur early in cultures of iPSC-derived DA neurons from PD patients, preceding the appearance of PDrelated morphological or functional phenotypes. Such methylation changes are not detected in undifferentiated iPSCs, nor in iPSC-derived neuronal cultures that are not enriched in DA neurons [41]. These results suggest that abnormalities in DNA methylation may underlie early disease manifestations in cell types vulnerable in PD. Future work will establish if targeting DNA methylation and its related pathways is a strategy that can be explored in combating neurodegeneration. A potential caveat of using iPSC-derived cultures to study disease-related epigenetic alterations is that the reprogramming processes may involve epigenomic reconfiguration of somatic cells $[47,48]$, which might confound the study of noncoding variants located at the hotspots for epigenomic reprogramming [47]. This should be taken into account for experimental design.

\section{Conclusion}

GWAS-identified risk variants hold important information for decoding molecular mechanisms of complex neurodegenerative diseases including AD and PD. Recently, human PSC-derived neuronal models have emerged as valuable tools for functional studies of non-coding GWAS variants. Breakthroughs in human microglia differentiation protocols have endowed us with the capacity of generating PSC-derived microglia and even combining them in $3 \mathrm{D}$ organoids to study immune-related $A D$ risk genes in vitro. Furthermore, genome-scale RNA-seq and epigenomic profiling of PSC-derived disease models represent new avenues 
for identifying gene or network perturbations associated with early disease status, which may hold promise for the development of new diagnostics and therapeutic strategies.

\section{Conflict of interest statement}

The authors declare no conflict of interest.

\section{Acknowledgements}

Support is provided by an ERC Starting Grant (260678), ERC Consolidator grant (646671), the Interuniversity Attraction Pole program by BELSPO, the research fund KU Leuven, a Methusalem grant of the Flemish government and VIB, Leuvens Universiteitsfonds (LUF) Opening the Future grant.

\section{References and recommended reading}

Papers of particular interest, published within the period of review, have been highlighted as:

*of special interest

** of outstanding interest

1. De Strooper B, Karran E: The Cellular Phase of Alzheimer's Disease. Cell 2016, 164:603-615.

2. de Lau LM, Breteler MM: Epidemiology of Parkinson's disease. Lancet Neurol 2006, 5:525-535.

3. Cuyvers $\mathrm{E}$, Sleegers K: Genetic variations underlying Alzheimer's disease: evidence from genomewide association studies and beyond. Lancet Neurol 2016, 15:857-868.

4. Lill CM: Genetics of Parkinson's disease. Mol Cell Probes 2016, 30:386-396.

5. Mattick JS: RNA regulation: a new genetics? Nat Rev Genet 2004, 5:316-323.

6. Rivera CM, Ren B: Mapping human epigenomes. Cell 2013, 155:39-55.

7. Young JE, Boulanger-Weill J, Williams DA, Woodruff G, Buen F, Revilla AC, Herrera C, Israel MA, Yuan SH, Edland SD, et al.: Elucidating molecular phenotypes caused by the SORL1 Alzheimer's disease genetic risk factor using human induced pluripotent stem cells. Cell Stem Cell 2015, 16:373-385.

8. Offe K, Dodson SE, Shoemaker JT, Fritz JJ, Gearing M, Levey AI, Lah JJ: The lipoprotein receptor LR11 regulates amyloid beta production and amyloid precursor protein traffic in endosomal compartments. J Neurosci 2006, 26:1596-1603.

9. Spoelgen R, von Arnim CA, Thomas AV, Peltan ID, Koker M, Deng A, Irizarry MC, Andersen OM, Willnow TE, Hyman BT: Interaction of the cytosolic domains of sorLA/LR11 with the amyloid precursor protein (APP) and beta-secretase beta-site APP-cleaving enzyme. J Neurosci 2006, 26:418-428.

10. Scherzer CR, Offe K, Gearing M, Rees HD, Fang G, Heilman CJ, Schaller C, Bujo H, Levey Al, Lah JJ: Loss of apolipoprotein E receptor LR11 in Alzheimer disease. Arch Neurol 2004, 61:1200-1205.

11. Soldner F, Stelzer Y, Shivalila CS, Abraham BJ, Latourelle JC, Barrasa MI, Goldmann J, Myers RH, Young RA, Jaenisch R: Parkinson-associated risk variant in distal enhancer of $\boldsymbol{\alpha}$-synuclein modulates target gene expression. Nature 2016, 533:95-99.

12. Ward LD, Kellis M: Interpreting noncoding genetic variation in complex traits and human disease. Nat Biotechnol 2012, 30:1095-1106. 
13. Kundaje A, Meuleman W, Ernst J, Bilenky M, Yen A, Heravi-Moussavi A, Kheradpour P, Zhang Z, Wang J, Ziller MJ, et al.: Integrative analysis of $\mathbf{1 1 1}$ reference human epigenomes. Nature 2015, 518:317-330.

14. Heppner FL, Ransohoff RM, Becher B: Immune attack: the role of inflammation in Alzheimer disease. Nat Rev Neurosci 2015, 16:358-372.

15. Zhang B, Gaiteri C, Bodea LG, Wang Z, McElwee J, Podtelezhnikov AA, Zhang C, Xie T, Tran L, Dobrin $\mathrm{R}$, et al.: Integrated systems approach identifies genetic nodes and networks in late-onset Alzheimer's disease. Cell 2013, 153:707-720.

16. Villegas-Llerena $C$, Phillips A, Garcia-Reitboeck P, Hardy J, Pocock JM: Microglial genes regulating neuroinflammation in the progression of Alzheimer's disease. Curr Opin Neurobiol 2016, 36:7481.

17. Muffat J, Li Y, Yuan B, Mitalipova M, Omer A, Corcoran S, Bakiasi G, Tsai LH, Aubourg P, Ransohoff $\mathrm{RM}$, et al.: Efficient derivation of microglia-like cells from human pluripotent stem cells. Nat Med 2016, 22:1358-1367.

18. Pandya H, Shen MJ, Ichikawa DM, Sedlock AB, Choi Y, Johnson KR, Kim G, Brown MA, Elkahloun AG, Maric $D$, et al.: Differentiation of human and murine induced pluripotent stem cells to microglia-like cells. Nat Neurosci 2017.

19. Abud EM, Ramirez RN, Martinez ES, Healy LM, Nguyen CHH, Newman SA, Yeromin AV, Scarfone VM, Marsh SE, Fimbres C, et al.: iPSC-Derived Human Microglia-like Cells to Study Neurological Diseases. Neuron 2017, 94:278-293.e279.

20. Fatehullah A, Tan SH, Barker N: Organoids as an in vitro model of human development and disease. Nat Cell Biol 2016, 18:246-254.

21. Lin L, Göke J, Cukuroglu E, Dranias MR, VanDongen AM, Stanton LW: Molecular Features Underlying Neurodegeneration Identified through In Vitro Modeling of Genetically Diverse Parkinson's Disease Patients. Cell Rep 2016, 15:2411-2426.

22. Chouhan AK, Guo C, Hsieh YC, Ye H, Senturk M, Zuo Z, Li Y, Chatterjee S, Botas J, Jackson GR, et al.: Uncoupling neuronal death and dysfunction in Drosophila models of neurodegenerative disease. Acta Neuropathol Commun 2016, 4:62.

23. Sánchez-Danés A, Richaud-Patin Y, Carballo-Carbajal I, Jiménez-Delgado S, Caig C, Mora S, Di Guglielmo C, Ezquerra M, Patel B, Giralt A, et al.: Disease-specific phenotypes in dopamine neurons from human iPS-based models of genetic and sporadic Parkinson's disease. EMBO Mol Med 2012, 4:380-395.

24. Israel MA, Yuan SH, Bardy C, Reyna SM, Mu Y, Herrera C, Hefferan MP, Van Gorp S, Nazor KL, Boscolo FS, et al.: Probing sporadic and familial Alzheimer's disease using induced pluripotent stem cells. Nature 2012, 482:216-220.

25. Kondo T, Asai M, Tsukita K, Kutoku Y, Ohsawa Y, Sunada Y, Imamura K, Egawa N, Yahata N, Okita K, et al.: Modeling Alzheimer's disease with iPSCs reveals stress phenotypes associated with intracellular $A \beta$ and differential drug responsiveness. Cell Stem Cell 2013, 12:487-496.

26. Chindelevitch L, Ziemek D, Enayetallah A, Randhawa R, Sidders B, Brockel C, Huang ES: Causal reasoning on biological networks: interpreting transcriptional changes. Bioinformatics 2012, 28:1114-1121.

27. Krämer A, Green J, Pollard J, Tugendreich S: Causal analysis approaches in Ingenuity Pathway Analysis. Bioinformatics 2014, 30:523-530.

28. Reinhardt P, Schmid B, Burbulla LF, Schöndorf DC, Wagner L, Glatza M, Höing S, Hargus G, Heck SA, Dhingra $A$, et al.: Genetic correction of a LRRK2 mutation in human iPSCs links parkinsonian neurodegeneration to ERK-dependent changes in gene expression. Cell Stem Cell 2013, 12:354367. 
29. Sandor C, Robertson P, Lang C, Heger A, Booth H, Vowles J, Witty L, Bowden R, Hu M, Cowley SA, et al.: Transcriptomic profiling of purified patient-derived dopamine neurons identifies convergent perturbations and therapeutics for Parkinson's disease. Hum Mol Genet 2017, 26:552-566.

30. Cannon JR, Tapias V, Na HM, Honick AS, Drolet RE, Greenamyre JT: A highly reproducible rotenone model of Parkinson's disease. Neurobiol Dis 2009, 34:279-290.

31. Sherer TB, Kim JH, Betarbet R, Greenamyre JT: Subcutaneous rotenone exposure causes highly selective dopaminergic degeneration and alpha-synuclein aggregation. Exp Neurol 2003, 179:916.

32. Finkelstein DI, Hare DJ, Billings JL, Sedjahtera A, Nurjono M, Arthofer E, George S, Culvenor JG, Bush Al, Adlard PA: Clioquinol Improves Cognitive, Motor Function, and Microanatomy of the AlphaSynuclein hA53T Transgenic Mice. ACS Chem Neurosci 2016, 7:119-129.

33. Kaur D, Yantiri F, Rajagopalan S, Kumar J, Mo JQ, Boonplueang R, Viswanath V, Jacobs R, Yang L, Beal $M F$, et al.: Genetic or pharmacological iron chelation prevents MPTP-induced neurotoxicity in vivo: a novel therapy for Parkinson's disease. Neuron 2003, 37:899-909.

34. Mazzio EA, Soliman KF: Basic concepts of epigenetics: impact of environmental signals on gene expression. Epigenetics 2012, 7:119-130.

35. Relton CL, Davey Smith G: Epigenetic epidemiology of common complex disease: prospects for prediction, prevention, and treatment. PLOS Med 2010, 7:e1000356.

36. De Jager PL, Srivastava G, Lunnon K, Burgess J, Schalkwyk LC, Yu L, Eaton ML, Keenan BT, Ernst J, McCabe $C$, et al.: Alzheimer's disease: early alterations in brain DNA methylation at ANK1, BIN1, RHBDF2 and other loci. Nat Neurosci 2014, 17:1156-1163.

37. Lunnon K, Smith R, Hannon E, De Jager PL, Srivastava G, Volta M, Troakes C, Al-Sarraj S, Burrage J, Macdonald R, et al.: Methylomic profiling implicates cortical deregulation of ANK1 in Alzheimer's disease. Nat Neurosci 2014, 17:1164-1170.

38. Masliah E, Dumaop W, Galasko D, Desplats P: Distinctive patterns of DNA methylation associated with Parkinson disease: identification of concordant epigenetic changes in brain and peripheral blood leukocytes. Epigenetics 2013, 8:1030-1038.

39. Davies MN, Volta M, Pidsley R, Lunnon K, Dixit A, Lovestone S, Coarfa C, Harris RA, Milosavljevic A, Troakes $C$, et al.: Functional annotation of the human brain methylome identifies tissuespecific epigenetic variation across brain and blood. Genome Biol 2012, 13:R43.

40. Roessler R, Smallwood SA, Veenvliet JV, Pechlivanoglou P, Peng SP, Chakrabarty K, Groot-Koerkamp MJ, Pasterkamp RJ, Wesseling E, Kelsey G, et al.: Detailed analysis of the genetic and epigenetic signatures of iPSC-derived mesodiencephalic dopaminergic neurons. Stem Cell Reports 2014, 2:520-533.

41. Fernández-Santiago R, Carballo-Carbajal I, Castellano G, Torrent R, Richaud Y, Sánchez-Danés A, Vilarrasa-Blasi R, Sánchez-Pla A, Mosquera JL, Soriano J, et al.: Aberrant epigenome in iPSCderived dopaminergic neurons from Parkinson's disease patients. EMBO Mol Med 2015, 7:1529-1546.

42. Stott SR, Metzakopian E, Lin W, Kaestner KH, Hen R, Ang SL: Foxa1 and foxa2 are required for the maintenance of dopaminergic properties in ventral midbrain neurons at late embryonic stages. J Neurosci 2013, 33:8022-8034.

43. Domanskyi A, Alter H, Vogt MA, Gass P, Vinnikov IA: Transcription factors Foxa1 and Foxa2 are required for adult dopamine neurons maintenance. Front Cell Neurosci 2014, 8:275.

44. Ros-Bernal F, Hunot S, Herrero MT, Parnadeau S, Corvol JC, Lu L, Alvarez-Fischer D, Carrillo-de Sauvage MA, Saurini F, Coussieu C, et al.: Microglial glucocorticoid receptors play a pivotal role in regulating dopaminergic neurodegeneration in parkinsonism. Proc Natl Acad Sci U S A 2011, 108:6632-6637. 
45. Santiago JA, Potashkin JA: Blood Biomarkers Associated with Cognitive Decline in Early Stage and Drug-Naive Parkinson's Disease Patients. PLoS One 2015, 10:e0142582.

46. Cao X, Yasuda T, Uthayathas S, Watts RL, Mouradian MM, Mochizuki H, Papa SM: Striatal overexpression of DeltaFosB reproduces chronic levodopa-induced involuntary movements. J Neurosci 2010, 30:7335-7343.

47. Lister R, Pelizzola M, Kida YS, Hawkins RD, Nery JR, Hon G, Antosiewicz-Bourget J, O'Malley R, Castanon $\mathrm{R}$, Klugman $\mathrm{S}$, et al.: Hotspots of aberrant epigenomic reprogramming in human induced pluripotent stem cells. Nature 2011, 471:68-73.

48. Kim K, Doi A, Wen B, Ng K, Zhao R, Cahan P, Kim J, Aryee MJ, Ji H, Ehrlich LI, et al.: Epigenetic memory in induced pluripotent stem cells. Nature 2010, 467:285-290. 